\title{
Target mass corrections revisited
}

\author{
F. M. Steffens ${ }^{1}$ and W. Melnitchouk ${ }^{2}$ \\ ${ }^{1}$ NFC-FCBEE-Universidade Presbiteriana Mackenzie, Rua da Consolação 930, 01302-907, São Paulo, SP, Brazil IFT-UNESP, \\ Rua Pamplona 145, 01405-900, São Paulo, SP, Brazil \\ ${ }^{2}$ Jefferson Lab, 12000 Jefferson Avenue, Newport News, Virginia 23606, USA
}

(Received 7 March 2006; published 8 May 2006)

\begin{abstract}
We propose a new implementation of target mass corrections to nucleon structure functions which, unlike existing treatments, has the correct kinematic threshold behavior at finite $Q^{2}$ in the $x \rightarrow 1$ limit. We illustrate the differences between the new approach and existing prescriptions by considering specific examples for the $F_{2}$ and $F_{L}$ structure functions, and discuss the broader implications of our results, which call into question the notion of universal parton distribution at finite $Q^{2}$.
\end{abstract}

DOI: 10.1103/PhysRevC.73.055202

PACS number(s): $13.60 . \mathrm{Hb}, 12.38 . \mathrm{Bx}$

\section{INTRODUCTION}

Deep inelastic lepton scattering is one of the most developed tools with which to probe the quark and gluon structure of hadrons. The theoretical framework within which the experiments are analyzed is the operator product expansion (OPE). This is well established in the Bjorken limit, which is defined by the four-momentum transfer squared $Q^{2}$ and energy transfer $v$ being asymptotically large, with the ratio $x=Q^{2} / 2 M v$ (the Bjorken scaling variable) fixed, where $M$ is the target mass. Within this framework, global analyses of deep inelastic scattering (DIS) and other experiments have allowed a vast array of data to be described in terms of a universal, process independent set of quark and gluon (or parton) distributions.

On the other hand, there exists a large body of data at lower energies, at $Q^{2} \lesssim 1-2 \mathrm{GeV}^{2}$ (see, e.g., Ref. [1]), where the use of the asymptotic, Bjorken limit formalism may be more questionable. In addition to perturbative QCD effects generated by gluon radiation, at low $Q^{2}$ effects arising from $1 / Q^{2}$ power corrections become increasingly important. These are typically generated by multiparton correlations, and within the twist expansion are associated with higher twists (in the OPE the twist of an operator is defined as its dimension minus its spin). Since they characterize the long-range nonperturbative interactions between quarks and gluons, the higher twists contain information on confinement dynamics, and are as such of intrinsic interest to study. Several recent analyses of structure function data at low $Q^{2}$ have extracted matrix elements of the higher twist operators [2].

Before one can reliably extract information on the higher twist contributions, it is important to remove from the data corrections arising from purely kinematic effects associated with finite values of $Q^{2} / v^{2}=4 M^{2} x^{2} / Q^{2}$. These so-called "target mass corrections" (TMCs) are formally related to twisttwo operators, and hence contain no additional information on nonperturbative multiparton correlations. Indeed, they have long been considered uninteresting and believed to be well understood. In the literature there are well known prescriptions for how to remove the TMC corrections $[3,4]$.

The TMCs were first considered by Nachtmann [5], who showed that one could arrange the OPE so as to ensure that at a given order in $1 / Q^{2}$ only operators of a given twist would appear. At finite $Q^{2}$ the natural scaling variable, defined as the fraction of the nucleon's light-front momentum $(p)$ carried by the parton $(k)$, is then given by the Nachtmann scaling variable:

$$
\xi\left(x, Q^{2}\right) \equiv \frac{k^{0}+k^{z}}{p^{0}+p^{z}}=\frac{2 x}{1+\sqrt{1+4 M^{2} x^{2} / Q^{2}}} .
$$

In the Nachtmann approach, one generalizes the CornwallNorton $(\mathrm{CN})$ moments of structure functions, derived in the Bjorken limit, to finite $Q^{2}$. A particular feature of these Nachtmann moments is that they are supposed to factor out the target mass dependence of the structure functions in a way such that its moments would equal the moments of the corresponding parton distributions.

Later Georgi and Politzer (GP) [3] calculated the $\mathrm{CN}$ moments of the structure functions, taking into account the trace terms which appear in the matrix elements of the twist-two operators, but which are usually neglected in high $Q^{2}$ data analyses. While the leading, twist-two piece of the structure function which enters at $\mathcal{O}(1)$ is related to matrix elements of the quark bilinear $\bar{\psi} \gamma^{\mu} \psi$, for example, the TMCs arise from insertions of derivatives, $\bar{\psi} \gamma^{\mu} D^{\mu_{1}} \cdots D^{\mu_{n}} \psi$, which does not alter the twist. This procedure generates a series in $M^{2} / Q^{2}$ when calculating the $\mathrm{CN}$ moments. Inverting these moments using the inverse Mellin transform, one arrives at a structure function depending on both $x$ and $M^{2} / Q^{2}$ [3].

Problems with the GP implementation of TMCs were soon identified, however, by a number of authors [6-10], in particular the so-called "threshold problem." This pertains to the fact that if the parton distribution function is a scaling function of $\xi$, then since the maximum kinematic value of $\xi$ at any finite $Q^{2}$ is $\xi_{0} \equiv \xi(x=1)<1$, the parton distribution is not defined in the unphysical region between the elastic limit $\xi=\xi_{0}$ and $\xi=1$. De Rujula et al. [11] argued that the problems can be resolved by considering in addition higher twist operators. They note that there is a nonuniformity in the limits as $n \rightarrow \infty$ and $Q^{2} \rightarrow \infty$, and the appearance of higher twist effects proportional to $n M^{2} / Q^{2}$ for the $n$th moment signals the breakdown of the entire approach at low $W(\lesssim 2 \mathrm{GeV})$. 
Tung and collaborators [8,9] attempted to redress the threshold problem by invoking an ansatz which smoothly merges the perturbative QCD behavior of the moments at large $Q^{2}$ with the correct threshold behavior in the $n \rightarrow \infty$ limit. As they note, however, such a prescription is not unique, and in fact agrees with the standard OPE expansion only in the $n \rightarrow \infty$ limit.

The proposed solution of De Rujula et al. [11] to the threshold problem implies that higher twist effects play an important role at low $Q^{2}$. Recent experiments at Jefferson Lab have shown, however, that the size of the higher twist contributions is actually quite small for the proton $F_{2}$ structure function, down to relatively low $Q^{2}$ values $\left(Q^{2} \sim 0.5-\right.$ $1 \mathrm{GeV}^{2}$ ) [12]. The question which we address here is whether a self-consistent formulation of TMCs can be made with only twist-two contributions, without appealing to higher twist effects. While not a proof, it seems plausible to us that, at least from a purely theoretical perspective, it should be possible to obtain an implementation of TMCs for a hypothetical case of negligible higher twist effects, which would demand a consistent resolution of the threshold problem for the twist-two part alone. Such a view could be motivated by observing that even though it is the same proton state that the twist-two and higher twist terms originate from, in principle the matrix elements of the local operators whose matrix elements characterize the twist expansion are in fact independent.

While interesting in its own right, the question of how to implement TMCs is also of practical importance, given the high quality electron-nucleon structure function data at low and moderate $Q^{2}$ which are being collected at Jefferson $\mathrm{Lab}$ [12]. TMCs are also vital in analyzing neutrino scattering data [13], much of which are taken at relatively low energies, and must be understood if one is to extract reliable information on neutrino oscillations for instance. For spin-dependent scattering, TMCs have also been calculated for the $g_{1}$ and $g_{2}$ structure functions [4], and recently for spin-1 targets such as the deuteron [14].

The pertinent question is whether the Nachtmann moment of the twist-two part of the structure function is $Q^{2}$ independent, as supposed in the original formulation [5]. In Sec. II we review the standard derivation and results for TMCs within the operator product expansion. We outline the problems associated with the standard approach, and suggest an alternative formulation designed to avoid the unphysical threshold problem. Earlier work [10] did indeed find that the Nachtmann moments do not account for all possible (leading twist) $M^{2} / Q^{2}$ effects. However, we suggest a prescription where the $M^{2} / Q^{2}$ dependence of the Nachtmann moments of the structure functions does equal, to very high accuracy, the $M^{2} / Q^{2}$ dependence of the moments of the quark distributions, for all $Q^{2}$. Numerical results are presented in Sec. III, where we compare the $x$ dependence of the $F_{2}$ and $F_{L}$ structure functions using the various TMC prescriptions, and examine the onset of scaling in terms of the Nachtmann moments of the structure functions. In Sec. IV we summarize our findings and discuss the broader implications of our results for the interpretation of parton distributions at finite $Q^{2}$.

\section{OPERATOR PRODUCT EXPANSION}

We begin this section by firstly reviewing the pioneering work on target mass corrections as obtained by Georgi and Politzer [3]. We will consider the case of unpolarized scattering from a spin-1/2 nucleon, which is described by two structure functions, $F_{1}\left(x, Q^{2}\right)$ and $F_{2}\left(x, Q^{2}\right)$ (or alternatively $F_{2}$ and the longitudinal structure function $F_{L}$ ). We shall focus on the $F_{2}$ structure function, but later generalize the discussion to include also $F_{L}$.

The two standard moments of structure functions encountered in the literature are the Cornwall-Norton and Nachtmann moments. The Cornwall-Norton moments of $F_{2}$ are given by

$$
M_{2}^{n}\left(Q^{2}\right)=\int_{0}^{1} d x x^{n-2} F_{2}\left(x, Q^{2}\right),
$$

and are appropriate for the region $Q^{2} \gg M^{2}$. The Nachtmann moments, on the other hand, take into account finite $M^{2} / Q^{2}$ corrections to the Bjorken limit, and are given by

$$
\begin{aligned}
\mu_{2}^{n}\left(Q^{2}\right)= & \int_{0}^{1} d x \frac{\xi^{n+1}}{x^{3}}\left[\frac{3+3(n+1) r+n(n+2) r^{2}}{(n+2)(n+3)}\right] \\
& \times F_{2}\left(x, Q^{2}\right)
\end{aligned}
$$

with $r=\sqrt{1+4 x^{2} M^{2} / Q^{2}}$. The essential difference between the $\mathrm{CN}$ and Nachtmann moments comes from the trace terms appearing in the matrix elements of operators of definite spin, which are disregarded in the $\mathrm{CN}$ approach, but kept in the Nachtmann approach. The Nachtmann moments are constructed such that from the infinite set of operators of twist-two and different spin contained in the trace terms, only the operators of $\operatorname{spin} n$ contribute for the $n-2$ moment of the structure function.

The Nachtmann and $\mathrm{CN}$ moments can be related by expanding the moments in powers of $1 / Q^{2}$. Expanding $\mu_{2}^{n}$ to $\mathcal{O}\left(1 / Q^{6}\right)$, one has

$$
\begin{aligned}
\mu_{2}^{n}\left(Q^{2}\right)= & M_{2}^{n}\left(Q^{2}\right)-\frac{n(n-1)}{n+2} \frac{M^{2}}{Q^{2}} M_{2}^{n+2}\left(Q^{2}\right) \\
& +\frac{n\left(n^{2}-1\right)}{2(n+3)} \frac{M^{4}}{Q^{4}} M_{2}^{n+4}\left(Q^{2}\right) \\
& -\frac{n\left(n^{2}-1\right)}{6} \frac{M^{6}}{Q^{6}} M_{2}^{n+6}+\cdots .
\end{aligned}
$$

Note that there is a mixing between the lower and higher moments. To this order we can also express the $\mathrm{CN}$ moments in terms of the Nachtmann moments:

$$
\begin{aligned}
M_{2}^{n}\left(Q^{2}\right)= & \mu_{2}^{n}\left(Q^{2}\right)+\frac{n(n-1)}{n+2} \frac{M^{2}}{Q^{2}} \mu_{2}^{n+2}\left(Q^{2}\right) \\
& +\frac{n\left(n^{2}-1\right)(n+2)}{2(n+3)(n+4)} \frac{M^{4}}{Q^{4}} \mu_{2}^{n+4}\left(Q^{2}\right) \\
& +\frac{n\left(n^{2}-1\right)(n+2)(n+3)}{6(n+5)(n+6)} \frac{M^{6}}{Q^{6}} \mu_{2}^{n+6}+\cdots .
\end{aligned}
$$

In the work of GP, the moment of the leading twist part of the $F_{2}$ structure function, corrected for target mass effects, can 
be written to order $1 / Q^{6}$ as

$$
\begin{aligned}
M_{n}^{\mathrm{GP}}\left(Q^{2}\right)= & A_{n}+\frac{n(n-1)}{n+2} \frac{M^{2}}{Q^{2}} A_{n+2} \\
& +\frac{n\left(n^{2}-1\right)(n+2)}{2(n+3)(n+4)} \frac{M^{4}}{Q^{4}} A_{n+4}\left(Q^{2}\right) \\
& +\frac{n\left(n^{2}-1\right)(n+2)(n+3)}{6(n+5)(n+6)} \frac{M^{6}}{Q^{6}} A_{n+6}+\cdots
\end{aligned}
$$

where $A_{n}$ is the $n$th moment of a distribution function $F(y)$ :

$$
A_{n}=\int_{0}^{y_{0}} d y y^{n} F(y) .
$$

Here the function $F(y)$ is related to the usual quark distribution $q(y)$ by $q(y) \equiv y F(y)$, and the upper limit of integration $y_{0}$ is the maximum value at which the quark distribution has physical support. Again in Eq. (6) there is a mixture between lower and higher moments. Comparing Eqs. (5) and (6), one can show that, at least to $\mathcal{O}\left(1 / Q^{6}\right)$, the Nachtmann moments are equivalent to the moments of the distribution $F(y)$ :

$$
\mu_{2}^{n} \equiv A_{n} \text {. }
$$

This reflects the fact that the Nachtmann moments are constructed to protect the moments of the structure functions from target mass effects, thereby allowing them to be identified directly with the moments of the quark distributions.

The $F_{2}$ structure function appearing in Eqs. (2) and (3) must itself be corrected for target mass effects, and to this end we will follow the procedure in GP [3], albeit with one exception. While GP write the upper limit of integration in Eq. (7) as $y_{0}=1$, we will define the upper limit of the integrals as the maximum value allowed by kinematics, $y_{0}=y(x=1)$. Following GP, we can then rewrite Eq. (7) as [3]

$$
\frac{A_{n+2 j}}{(n+2 j)(n+2 j-1)}=\int_{0}^{y_{0}} d y y^{n+2 j-2} G(y),
$$

with $G(y)$ given by

$$
G(y)=\int_{y}^{y_{0}} d y^{\prime} H\left(y^{\prime}\right)=\int_{y}^{y_{0}} d y^{\prime} \int_{y^{\prime}}^{y_{0}} d y^{\prime \prime} F\left(y^{\prime \prime}\right) .
$$

This result follows from the fact that

$$
\begin{aligned}
\int_{0}^{y_{0}} d y y^{n+2 j-2} G(y)= & \left.\frac{y^{n+2 j-1}}{n+2 j-1} G(y)\right|_{0} ^{y_{0}} \\
& -\int_{0}^{y_{0}} d y \frac{y^{n+2 j-1}}{n+2 j-1} \frac{\partial G(y)}{\partial y},
\end{aligned}
$$

and because $G(0)=G\left(y_{0}\right)=0$, one is left with the second term only. Integrating the RHS of Eq. (11) again, we recover Eq. (9). To obtain the $x$ (or $\xi$ ) dependence of the structure functions, we can invert the moment as in GP, arriving at same results but with a modified upper limit in the integrals. Specifically, we recover for the $F_{2}$ structure function [3]

$$
\begin{aligned}
F_{2}\left(x, Q^{2}\right)= & \frac{\xi^{2}\left(1-a^{2} \xi^{2}\right)}{\left(1+a^{2} \xi^{2}\right)^{3}} F(\xi)+6 a^{2} \frac{\xi^{3}\left(1-a^{2} \xi^{2}\right)}{\left(1+a^{2} \xi^{2}\right)^{4}} H(\xi) \\
& +12 a^{4} \frac{\xi^{4}\left(1-a^{2} \xi^{2}\right)}{\left(1+a^{2} \xi^{2}\right)^{5}} G(\xi)
\end{aligned}
$$

where $a \equiv M / Q$. The $\mathrm{CN}$ moments of the target mass corrected $F_{2}$ structure function are then given by [3]

$$
\begin{aligned}
M_{2}^{n}\left(Q^{2}\right) & =\int_{0}^{1} d x x^{n-2} F_{2}\left(x, Q^{2}\right) \\
& =\sum_{j=0}^{\infty}\left(\frac{M^{2}}{Q^{2}}\right)^{j} \frac{(n+j) !}{j !(n-2) !} \frac{A_{n+2 j}}{(n+2 j)(n+2 j-1)} .
\end{aligned}
$$

To calculate the Nachtmann moments, we rewrite Eq. (3) in terms of $\xi$ :

$$
\begin{aligned}
\mu_{2}^{n}\left(Q^{2}\right)= & \int_{0}^{\xi_{0}} d \xi \xi^{n-2} \frac{\left(1+a^{2} \xi^{2}\right)^{3}}{1-a^{2} \xi^{2}} F_{2}\left(\xi, Q^{2}\right) \\
& \times\left[1-\frac{3(r-1)}{r^{2}(n+2)}-\frac{3(r-1)^{2}}{r^{2}(n+3)}\right],
\end{aligned}
$$

with $F_{2}\left(\xi, Q^{2}\right)$ given by Eq. (12), and

$$
\xi_{0}=\xi(x=1)=\frac{2}{1+\sqrt{1+4 M^{2} / Q^{2}}} .
$$

In the following section we will examine the extent to which the Nachtmann moments of $F_{2}$ correspond to the moments $A_{n}$ of the quark distribution function, for different functional forms of $F(\xi)$, and quantify the effect of the kinematic thresholds. Central will be the interpretation of the function $F(\xi)$ itself.

Before proceeding, for completeness we also give the results for the longitudinal structure function, $F_{L}$, and its moments. In the $Q^{2} \rightarrow \infty$ limit, $F_{L}=0$, while at finite $Q^{2}$ the TMCs render $F_{L}$ nonzero. Of course, higher order perturbative QCD corrections which depend on $\alpha_{s}$ also give rise to a nonzero $F_{L}$, as do higher twist effects. However, we artificially set both of these to zero in order to isolate the effects of TMCs on $F_{L}$ explicitly. Following a similar procedure as for $F_{2}$ above, we can write the longitudinal structure function as

$$
F_{L}\left(\xi, Q^{2}\right)=\frac{2 a^{2} \xi^{2}}{\left(1+a^{2} \xi^{2}\right)^{2}} H(\xi)+\frac{4 a^{4} \xi^{3}}{\left(1+a^{2} \xi^{2}\right)^{3}} G(\xi),
$$

which clearly vanishes as $a \rightarrow 0$ (or $Q^{2} \rightarrow \infty$ ). The corresponding Nachtmann moments are then given by

$$
\begin{aligned}
\mu_{L}^{n}\left(Q^{2}\right)= & \int_{0}^{\xi_{0}} d \xi \xi^{n-2}\left(1-a^{4} \xi^{4}\right)\left(F_{L}\left(\xi, Q^{2}\right)+\frac{4 a^{2} \xi^{2}}{\left(1-a^{2} \xi^{2}\right)}\right. \\
& \left.\times \frac{(n+1)\left(1-a^{2} \xi^{2}\right)-2(n+2)}{(n+2)(n+3)} F_{2}\left(\xi, Q^{2}\right)\right) .
\end{aligned}
$$

Having derived analytic expressions for the $F_{2}$ and $F_{L}$ structure functions and their moments, in the next section we present numerical results using several different prescriptions for the $\xi$ dependence of the quark distribution function.

\section{TARGET MASS CORRECTIONS}

The main purpose of this work is to analyze phenomenologically what is the best procedure to incorporate TMCs in the analysis of structure functions. More specifically, we address the question of which procedure is most effective in 
rendering the moments of the leading twist structure functions equal to the moments of the quark distributions at finite $Q^{2}$. We consider whether there is any sizable difference for the moments when the upper limit of the integrals in $G(y)$ and $H(y)$ is 1 or $\xi_{0}$. In particular, since the twist-two part of the deep inelastic cross section should be zero at $x=1\left(\xi=\xi_{0}\right)$, we study the impact of a vanishing parton distribution at $\xi_{0}$.

\section{A. Prescriptions}

To address these issues, in this section we present several prescriptions for the implementation of target mass corrections, and discuss their limitations and practical consequences. We consider three scenarios:

(A) Integrate a quark distribution:

$$
q(\xi)=\mathcal{N} \xi^{-1 / 2}(1-\xi)^{3}
$$

from 0 to 1 (specifically, in the integrals for $A_{n}, H(\xi)$ and $G(\xi))$. Here the normalization $\mathcal{N}$ ensures that the distribution integrates to unity. We denote this prescription the "standard TMC" (sTMC).

(B) Integrate a modified distribution which vanishes for $\xi>\xi_{0}$, as implied by Eq. (7): ${ }^{1}$

$$
q(\xi)=\mathcal{N} \xi^{-1 / 2}(1-\xi)^{3} \Theta\left(\xi-\xi_{0}\right) .
$$

We denote this prescription the "modified TMC" (mTMC).

(C) Use a "threshold dependent" (TD) quark distribution which vanishes in the physical limit:

$$
q^{\mathrm{TD}}(\xi)=\mathcal{N} \xi^{-1 / 2}\left(\xi_{0}-\xi\right)^{3} .
$$

Note that because of the upper limit in Eq. (7), $A_{n}$ itself will be $M^{2} / Q^{2}$ dependent for prescriptions $\mathrm{B}$ and $\mathrm{C}$. The results for the ratio $\mu_{2}^{n} / A_{n}$ of the $n=2$ moments are displayed in Fig. 1 for the three cases, with prescriptions $\mathrm{A}, \mathrm{B}$ and

${ }^{1}$ We believe this was also the implication of De Rújula et al. [11].

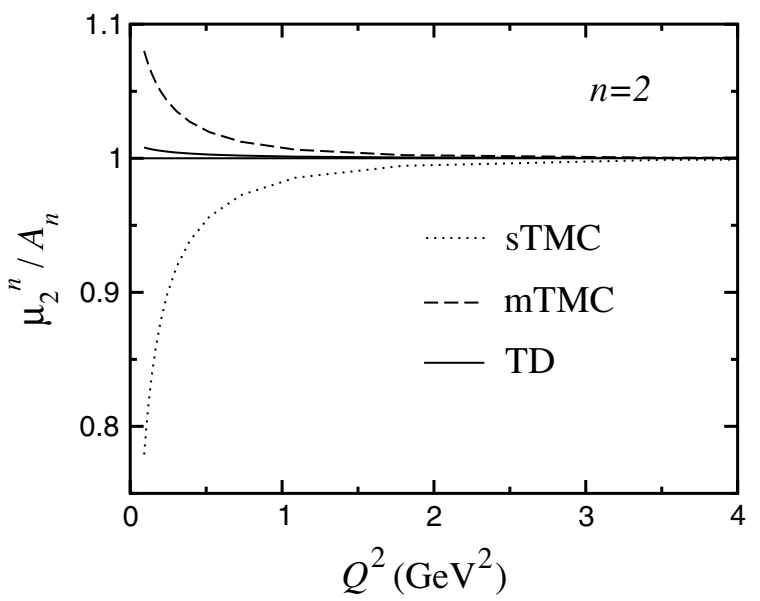

FIG. 1 . Ratio of the $n=2$ Nachtmann moment of the $F_{2}$ structure function and the $n=2$ moment of the quark distribution, as a function of $Q^{2}$. The curves correspond to prescriptions A ["sTMC"] (dotted), B ["mTMC"] (dashed) and C [“TD"] (solid).
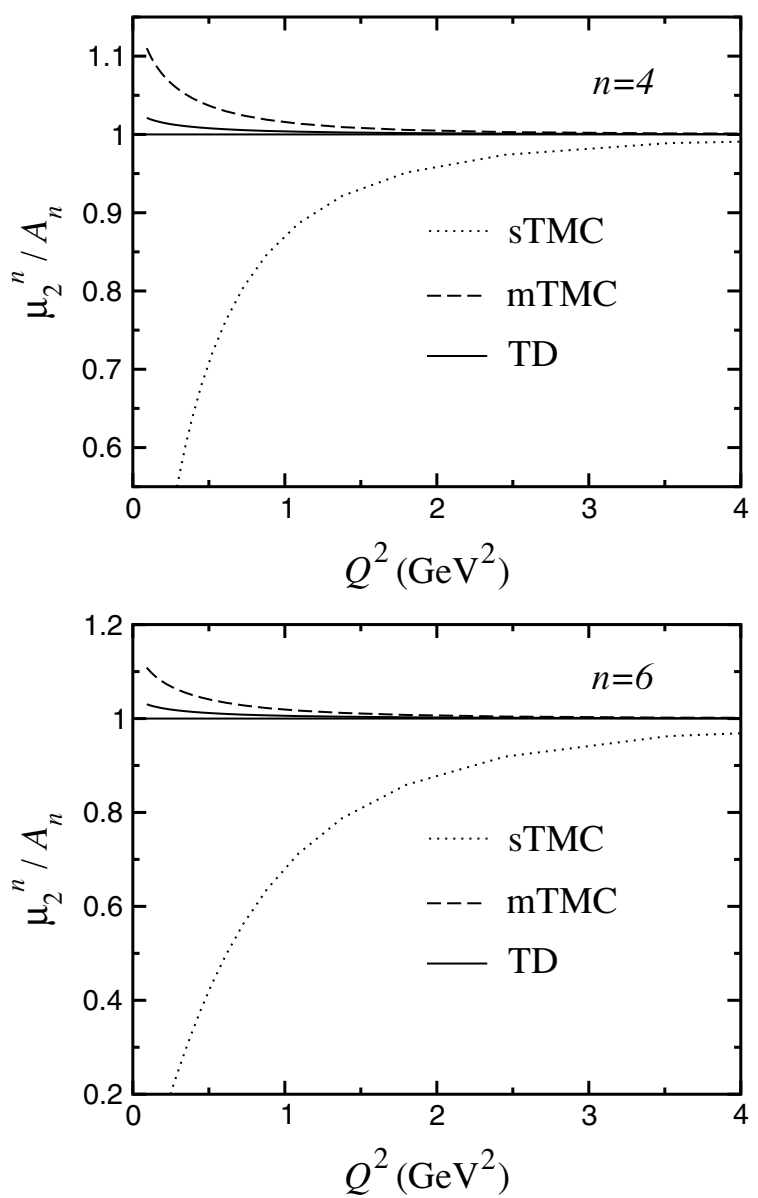

FIG. 2. Ratios of the $n=4$ (upper graph) and $n=6$ (lower graph) Nachtmann moment of the $F_{2}$ structure function and the corresponding moments of the quark distribution, as a function of $Q^{2}$. The curves are as in Fig. 1 .

C corresponding to the dotted, dashed and solid curves, respectively. Comparing the sTMC and $\mathrm{mTMC}$ results, one can see a reduced $Q^{2}$ dependence when the integrals are restricted to $\xi<\xi_{0}$. However, a much more dramatic change occurs when the quark distribution is constrained to vanish at $\xi_{0}$. This renders the Nachtmann moment almost equal to the moment of the quark distribution for virtually all $Q^{2}$ considered. Certainly for $Q^{2}>1 \mathrm{GeV}^{2}$ there is no visible deviation of the ratio from unity. Even for very small $Q^{2}, Q^{2} \sim 0.3 \mathrm{GeV}^{2}$, the ratio differs from unity by only $\sim 0.7 \%$ (of course the OPE itself may not be valid at such low values of $Q^{2}$ ).

Similarly, the ratios for the $n=4$ and $n=6$ moments are shown in Fig. 2. The deviation of the ratio from unity for the sTMC approach is between $10 \%-20 \%$ for $Q^{2} \lesssim 1 \mathrm{GeV}^{2}$, while that for the modified TMC with prescription $\mathrm{B}$ is of the order of $5 \%$ over the same $Q^{2}$ region. On the other hand, for the threshold dependent prescription $\mathrm{C}$, the deviation from unity remains around $1 \%$ even at these low $Q^{2}$ values.

A consequence of prescription $\mathrm{C}$ is that the moments of the parton distribution are $Q^{2}$ dependent. This seems to be an inevitable consequence if the Nachtmann moments of the structure function are to be equal to the moments of the parton distribution for all $Q^{2}$. Note that this $Q^{2}$ dependence is not of 
higher twist or perturbative QCD origin, but arises solely from kinematics. Nevertheless, this avoids the more serious problems which arise within the sTMC approach (prescription A), where the Nachtmann moments below $Q^{2} \sim 1 \mathrm{GeV}^{2}$ start to deviate significantly from the moments of the quark distributions. In addition, in the sTMC formulation one is faced with the so-called "threshold problem." Namely, if the moments $A_{n}$ of the quark distributions are $Q^{2}$ independent, then one should have

$$
\int_{0}^{1} d \xi \xi^{n} F\left(\xi, Q_{1}^{2}\right)=\int_{0}^{1} d \xi \xi^{n} F\left(\xi, Q_{2}^{2}\right)
$$

for any two momentum scales $Q_{1}^{2}$ and $Q_{2}^{2}$. Since $F\left(\xi, Q^{2}\right)$ must vanish in the kinematically forbidden region $\xi>\xi_{0}$, the equality in Eq. (21) implies that the function must be zero for both $\xi>\xi_{0}\left(Q_{1}^{2}\right)$ and $\xi>\xi_{0}\left(Q_{2}^{2}\right)$. If $Q_{1}^{2}<Q_{2}^{2}$, in which case $\xi_{0}\left(Q_{1}^{2}\right)<\xi_{0}\left(Q_{2}^{2}\right)$, this implies that $F\left(\xi, Q_{2}^{2}\right)$ should vanish in the range $\xi_{0}\left(Q_{1}^{2}\right)<\xi<\xi_{0}\left(Q_{2}^{2}\right)$. However, there is no physical reason for it to do so here, and this therefore leads to an unphysical constraint.

De Rújula et al. [11] address this problem by pointing out that higher twist contributions play an ever more important role at low $Q^{2}$, and their neglect makes any leading twist analysis at large $\xi$ incomplete. On the other hand, the philosophy inherent in prescription $C$ is that the equality (21) should not be expected to hold, simply because the $\xi$ dependence, and also the normalization, of the quark distributions is $M^{2} / Q^{2}$ dependent:

$$
\int_{0}^{\xi_{0}\left(Q_{1}^{2}\right)} d \xi \xi^{n} F\left(\xi, Q_{1}^{2}\right) \neq \int_{0}^{\xi_{0}\left(Q_{2}^{2}\right)} d \xi \xi^{n} F\left(\xi, Q_{2}^{2}\right) .
$$

Another approach to dealing with the threshold problem, as discussed in Ref. [14] for example, is to expand the TMC corrected structure functions in Eqs. (12) and (16) in a power series in $1 / Q^{2}$. Keeping only the leading $\mathcal{O}\left(1 / Q^{2}\right)$ correction, one finds that the corrected structure function vanishes in the $x \rightarrow 1$ limit, although in principle higher order corrections will become more important as $x \rightarrow 1$.

In the following section we will contrast the various prescriptions by studying their effects on the structure functions numerically.

\section{B. Numerical results for structure functions}

The effects of TMCs on the $F_{2}$ structure function are illustrated in Fig. 3 for $Q^{2}=1$ and $5 \mathrm{GeV}^{2}$ for the various scenarios. Here the scaling function $x q(x)$ (dotted curve) is used as input in Eq. (12) to calculate the target mass corrected function $F_{2}$. To translate the results from $\xi$ to $x$, we fix $Q^{2}$ and extract the corresponding $x$ for each $\left(\xi, Q^{2}\right)$ pair. For the sTMC method (prescription A, dashed), in which the upper limits of the integrals are set to unity, the corrected structure function becomes smaller at intermediate $x$ values, but larger as $x \rightarrow 1$. The corrected structure function for the mTMC approach (prescription B, double-dot-dashed), where the $\xi$ integration is constrained by $\xi<\xi_{0}$, display a similar behavior as a function of $x$. In both cases $F_{2}$ is clearly nonzero in the $x \rightarrow 1$ limit. The effects are sizable at $Q^{2}=1 \mathrm{GeV}^{2}$, but
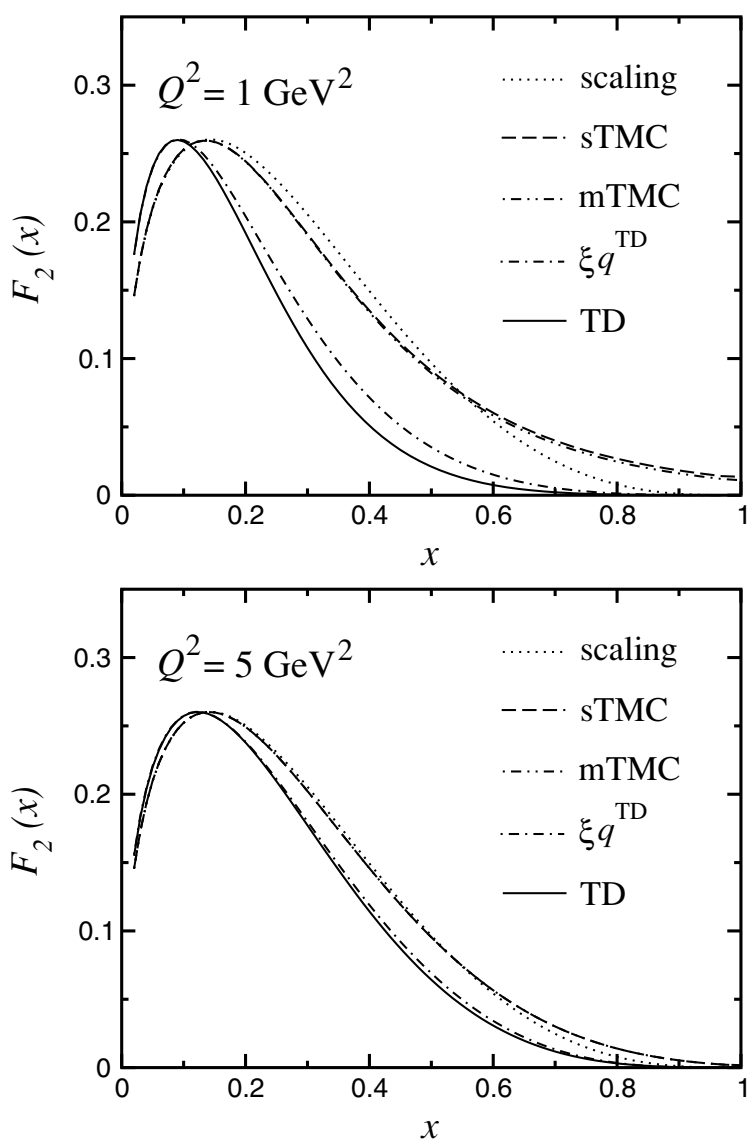

FIG. 3. The $x$ dependence of the $F_{2}$ structure function at $Q^{2}=$ $1 \mathrm{GeV}^{2}$ (upper) and $5 \mathrm{GeV}^{2}$ (lower). The effects of TMCs on the (input) scaling distribution (dotted curve) are illustrated for the sTMC (dashed) and mTMC (double-dot-dashed) prescriptions, and compared with the effects on the (input) TD-distribution $\xi q^{\mathrm{TD}}(\xi)$ (dot-dashed) using the TD approach (prescription C, solid).

considerably smaller at $Q^{2}=5 \mathrm{GeV}^{2}$, where the differences between the scaling and target mass corrected functions are more strongly suppressed. At lower $Q^{2}$ values, $Q^{2} \sim$ $0.5 \mathrm{GeV}^{2}$ (not shown), the differences between the sTMC and mTMC prescriptions are even more pronounced, so that here it is important to take into account the correct kinematics, especially at large $x$. Note that the sTMC and mTMC curves in Fig. 3 were normalized such that the quark number at finite $Q^{2}$ is equal to the quark number at $Q^{2} \rightarrow \infty$.

The effect of the threshold dependent prescription $\mathrm{C}$ is dramatically different from the other prescriptions. Specifically, the TD input distribution $q^{\mathrm{TD}}$ (dot-dashed curve in Fig. 3) produces a target mass corrected leading twist structure function which is exactly zero at $x=1\left(\xi=\xi_{0}\right)$ for all $Q^{2}$, as required physically. Another important difference between the sTMC and mTMC approaches, and the TD prescription, is at intermediate $x$. Here the latter produces a corrected structure function which is smaller than the input, in contrast with the sTMC/mTMC methods, where the corrected $F_{2}$ is larger than the input scaling function. Such differences may be very relevant in phenomenological determinations of the $x$ dependence of parton distributions at low $Q^{2}$. 

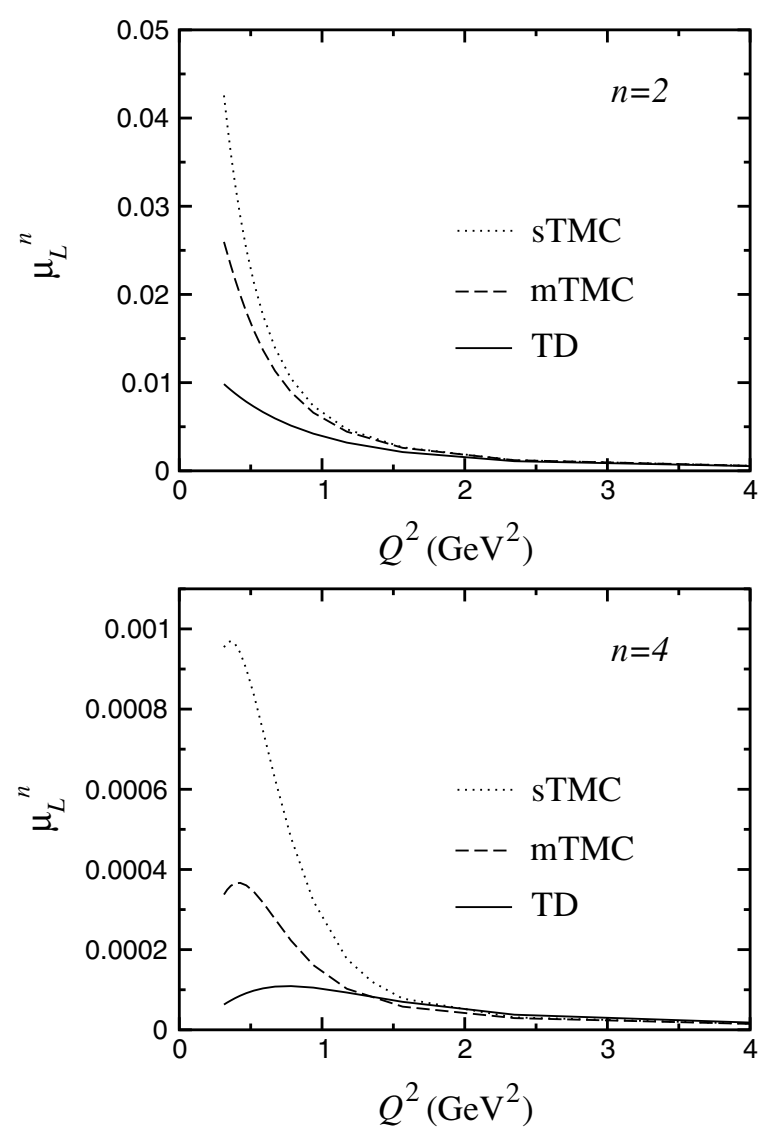

FIG. 4. Nachtmann moments of the longitudinal structure function for $n=2$ (upper) and $n=4$ (lower) as a function of $Q^{2}$.

How does the TD prescription $\mathrm{C}$ help in the practical extraction of leading twist parton distributions? If we define

$$
A_{n} \equiv \xi_{0}^{-(n+3 / 2)} A_{n}\left(Q^{2}\right),
$$

then since at high $Q^{2}$ the moments of the quark distributions become $Q^{2}$ independent, so too are the Nachtmann moments of the structure functions, as the multiplicative factor is $\xi$ independent. Other prescriptions would render a different $\xi_{0}$, but the important fact is that once $\mu_{2}^{n}\left(Q^{2}\right)=A_{n}\left(Q^{2}\right)$ for any $Q^{2}$, this equality may be set to its value at $Q^{2} \rightarrow \infty$ :

$$
\frac{\mu_{2}^{n}\left(\text { finite } Q^{2}\right)}{A_{n}\left(\text { finite } Q^{2}\right)}=\frac{\mu_{2}^{n}\left(Q^{2} \rightarrow \infty\right)}{A_{n}\left(Q^{2} \rightarrow \infty\right)}=\frac{M_{2}^{n}}{A_{n}} .
$$

This makes the approach in prescription $\mathrm{C}$ much more useful for extracting quark distributions from structure function data at low $Q^{2}$.

A comparison of the different TMC prescriptions for the longitudinal structure function moments is shown in Fig. 4, for the $n=2$ and $n=4$ Nachtmann moments. Here we plot the absolute value of the moments rather than a ratio, since the moments in the scaling limit are identically zero. For the sTMC prescription, the corrected moments are strongly $Q^{2}$ dependent for $Q^{2} \lesssim 1 \mathrm{GeV}^{2}$ and rise rapidly as $Q^{2}$ decreases. The $Q^{2}$ dependence of the moments for the mTMC approach, with the upper integration limit being $\xi_{0}$ rather than unity, is

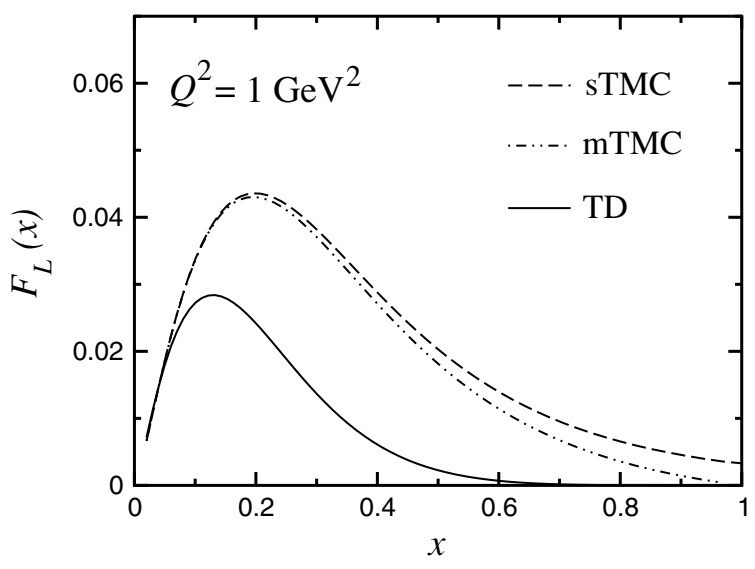

FIG. 5. Longitudinal structure function $F_{L}$ at $Q^{2}=1 \mathrm{GeV}^{2}$ for the sTMC (dashed), mTMC (double-dot-dashed) and TD-distribution (solid) prescriptions. Note that the scaling longitudinal distribution is zero.

somewhat weaker, but still quite strong at low $Q^{2}$. On the other hand, the moments for the TD prescription $\mathrm{C}$ display significantly smaller $Q^{2}$ dependence at low $Q^{2}$.

Finally, for completion, we show in Fig. 5 the $F_{L}$ structure function at $Q^{2}=1 \mathrm{GeV}^{2}$ with TMCs applied according to the three prescriptions above. Note that in the scaling limit, the $F_{L}$ structure function is identically zero. For the sTMC and $\mathrm{mTMC}$ prescriptions, the corrected structure function is significantly larger in magnitude than for the TD prescription at intermediate and large $x$. For the sTMC case in particular, it is also seen to approach a nonzero value in the $x \rightarrow 1$ limit. This result suggests that the evaluation of the twist-two part of the longitudinal structure function at low $Q^{2}$ may also need to be reassessed in phenomenological analyses, especially at intermediate and large $x$.

\section{CONCLUSION}

In this work we have revisited the long-standing problem of target mass corrections to nucleon structure functions. The standard procedure for implementing target mass effects suffers from the well known threshold problem, in which the corrected, leading twist structure function does not vanish at $x=1$. We have proposed a solution to this problem by introducing a finite- $Q^{2}$, "threshold dependent" parton distribution function that explicitly depends on the kinematical threshold $\xi_{0}$, which is smooth in the entire physical region, and approaches the ordinary, $Q^{2}$-independent parton distribution in the limit $Q^{2} \rightarrow \infty$. Our prescription avoids any discontinuities in the parton distributions and structure functions at finite $Q^{2}$, and produces vanishing structure functions as $x \rightarrow 1$. This is true for both the $F_{2}$ and $F_{L}$ structure functions.

The Nachtmann moments $\mu_{2}^{n}$ of the $F_{2}$ structure function, calculated with the threshold dependent distributions $q^{\mathrm{TD}}$, agree with the moments $A_{n}$ of $q^{\mathrm{TD}}$ to within $1 \%$ for the $n=2,4$ and 6 moments for $Q^{2}$ as low as $1 \mathrm{GeV}^{2}$ and even lower. In contrast, the deviation for the standard or modified TMC procedure (sTMC or mTMC prescriptions) is more than 
an order of magnitude larger at the same $Q^{2}$ values, and grows rapidly with increasing $n$. Furthermore, for $Q^{2}>M^{2}$ one can show analytically that, at least to $\mathcal{O}\left(1 / Q^{6}\right)$, the moments $\mu_{2}^{n}$ and $A_{n}$ are identical. Similarly, for the longitudinal structure function $F_{L}$, the Nachtmann moments $\mu_{L}^{n}$ with the threshold dependent distribution are considerably smaller (i.e. closer to the asymptotic value of zero) than the moments in the sTMC or mTMC prescriptions.

A consequence of our formulation is that the moments of the threshold dependent distributions will in general be $M^{2} / Q^{2}$ dependent. This dependence is not associated with either perturbative QCD effects or higher twists, but comes entirely from the leading twist, target mass effects. Our analysis suggests that it may be necessary to reassess the interpretation of a parton distribution in the presence of the finite $M^{2} / Q^{2}$, or $\xi$, corrections, as well as the implementation of the $q^{\mathrm{TD}}$ distributions in the $Q^{2}$ evolution equations. We will address these problems in future work [15]. At the same time, our numerical results give impetus to investigating the impact of TMCs on phenomenological fits to structure functions at low $Q^{2}[16]$ and the extraction of twist-two parton distributions.

\section{ACKNOWLEDGMENTS}

We would like to thank J. Blümlein, M. E. Christy, C. E. Keppel, and A. W. Thomas for helpful discussions. This work was supported by the U.S. Department of Energy contract DEAC05-84ER40150, under which the Southeastern Universities Research Association (SURA) operates the Thomas Jefferson National Accelerator Facility (Jefferson Lab). F.M.S. is also supported by FAPESP (03/10754-0) and CNPq (308932/ 2003-0).
[1] W. Melnitchouk, R. Ent, and C. Keppel, Phys. Rep. 406, 127 (2005)

[2] Z.-E. Meziani, W. Melnitchouk, J.-P. Chen, S. Choi et al., Phys. Lett. B613, 148 (2005); M. Osipenko et al., ibid. B609, 259 (2005); A. Deur et al., Phys. Rev. Lett. 93, 212001 (2004); M. Osipenko et al., Phys. Rev. D 71, 054007 (2005); S. I. Alekhin, S. A. Kulagin, and S. Liuti, ibid. 69, 114009 (2004); N. Bianchi, A. Fantoni, and S. Liuti, ibid. 69, 014505 (2004); M. Osipenko, W. Melnitchouk, S. Simula, S. Kulagin, and G. Ricco, Nucl. Phys. A766, 142 (2006).

[3] H. Georgi and H. D. Politzer, Phys. Rev. D 14, 1829 (1976).

[4] J. Blümlein and A. Tkabladze, Nucl. Phys. B553, 427 (1999); J. Phys. G 25, 1553 (1999).

[5] O. Nachtmann, Nucl. Phys. B63, 237 (1973).

[6] R. K. Ellis, R. Petronzio, and G. Parisi, Phys. Lett. B64, 97 (1976); R. Barbieri, J. R. Ellis, M. K. Gaillard, and G. G. Ross, ibid. B64, 171 (1976); Nucl. Phys. B117, 50 (1976).
[7] D. J. Gross, S. B. Treiman, and F. A. Wilczek, Phys. Rev. D 15, 2486 (1977).

[8] K. Bitar, P. W. Johnson, and W. K. Tung, Phys. Lett. B83, 114 (1979).

[9] P. W. Johnson and W. K. Tung, Print-79-1018 (Illinois Tech), Contribution to Neutrino '79, Bergen, Norway, 18-22 June 1979.

[10] W. R. Frazer and J. F. Gunion, Phys. Rev. Lett. 45, 1138 (1980).

[11] A. De Rujula, H. Georgi, and H. D. Politzer, Phys. Rev. D 15, 2495 (1977).

[12] I. Niculescu et al., Phys. Rev. Lett. 85, 1182 (2000); 85, 1186 (2000).

[13] S. Kretzer and M. H. Reno, Phys. Rev. D 69, 034002 (2004).

[14] S. A. Kulagin and R. Petti, Nucl. Phys. A765, 126 (2006).

[15] F. M. Steffens et al. (in preparation).

[16] M. E. Christy et al. (private communication). 